\title{
Editorial
}

\section{The Immune System and Complementary and Alternative Medicine}

\author{
Edwin L. Cooper \\ Laboratory of Comparative Neuroimmunology, Department of Neurobiology, David Geffen School of Medicine \\ at UCLA, University of California, Los Angeles, Los Angeles California 90095-1763, USA
}

Keywords: alternative medicine - complementary medicine - immune system - NK cells

\section{Introduction}

Some may wonder why a comparative immunologist would knock on the door of the controversial field of complementary and alternative medicine (CAM). The field is replete with problems associated with beliefs, disbeliefs, shaky concepts and ill-defined experimental approaches at solving fundamental questions. Yet there is a measure of order in that miniscule, infinitesimal corner of phenomena that begs for clarification and solid attempts to create order from the chaos. Such was the world of comparative immunology in the early 60 s when the parent field of immunology was budding into the giant sequoia of a discipline that it is today. There was essentially one model the eternal mouse whose spleen seemed to provide the essence of immunology! How did it all get started and how did that somewhat fortuitous birth provide succor for a field that could approach CAM. Entering CAM with an evidence-based approach was therefore as much of a fruitful and fulfilling challenge as trying to probe convincingly the existence of innate immunity in earthworms $(1,2)$.

\section{Are There Really Linkages between Immunity and CAM? See Perspectives and Admonitions}

Invertebrates possess elements of both non-specific and specific immune responses. Although these concepts have been subjected to intense scrutiny, these two elements are often controversial. One point of contention, perhaps

For reprints and all correspondence: Edwin L. Cooper, Laboratory of Comparative Neuroimmunology, Department of Neurobiology, David Geffen School of Medicine at UCLA, University of California,

Los Angeles, Los Angeles California 90095-1763. Tel: (310) 825-9567;

Fax: (310) 825-2224; E-mail: cooper@mednet.ucla.edu the chief, may be traced to the earliest history of immunology that was rooted first in the humoral system. A commonly held belief concerning the immune system is that its progressive, phylogenetic development paralleled the appearance of immunoglobulins and T-cell receptors (TCR) in vertebrates. If this is true, the entire animal kingdom can be divided according to this narrow view: (i) invertebrates that produce only nonspecific reactions by leukocytes and humoral components; (ii) vertebrates that additionally show specific responses through $\mathrm{T}$ cells and $\mathrm{B}$ cells that destroy cancers and synthesize immunoglobulins. Because the cell-mediated immune response appears to be prototypic, it may deserve newer approaches in order to reveal increasing and progressive levels of differentiation, especially when invertebrates are considered. Thus strict demarcations seem blurred.

With respect to immunity, there has always been the dominant anthropocentric theme starting most vividly at the end of the 18th century with Jenner's attempts at vaccination. No one really investigated the universality of mechanisms or the possible existence or at least importance of beings other than humans, not at least with respect to immunology. However, the current status of immunity can be described using two general terms: cellular immunity and humoral immunity. These two great camps are in turn subdivided into innate immunity and adaptive immunity. Innate immunity is characteristically non-specific, natural non-anticipatory, non-clonal and germline. On the other hand, adaptive immunity is specific, induced anticipatory, clonal and somatic. Each of these terms defines particular attributes and when compared represents distinct underlying mechanisms. Invertebrates are considered to possess cells and molecules that almost exclusively effect only innate responses.

This is an Open Access article distributed under the terms of the Creative Commons Attribution Non-Commercial License (http://creativecommons.org/ licenses/by-nc/2.0/uk/) which permits unrestricted non-commercial use, distribution, and reproduction in any medium, provided the original work is properly cited. 
Vertebrates retained this innate response but also evolved the adaptive response.

\section{Early Roots of Immunity: Planting the Seeds for CAM?}

Metchnikoff's contribution was also instrumental in forging the discipline of comparative immunology, a branch of immunology that investigates the evolution of immune mechanisms by experimentation using different animal species. Invertebrate immunology is considered a subdiscipline of this since it deals with several related features. First, there is the need to understand the basis of innate, natural, non-specific, non-anticipatory and non-clonal responses, which is the only response of invertebrates according to current evidence. Second, a thorough understanding of diverse mechanisms is essential if we are to define the various steps in the evolutionary development of the invertebrate type of response and its relationship to that of vertebrates. In addition to this invertebrate immune system, with progressive and more complicated evolutionary steps, a more intricate system appeared in vertebrates: the highly evolved and presumably more advanced adaptive, induced, specific, anticipatory and clonal responses. Third, invertebrate immune systems are of interest as experimental subjects because, by revealing their immune response characteristics, we can better comprehend environmental forces that may have influenced the evolution of immune systems. Clearly the most prominent force was probably the survival of a species by virtue of having evolved an immune system $(2,3)$.

\section{Arrival at the Door of CAM: Immunology Has The Key?}

Now from a famous editorial for the journal, Evidence Based Complementary and Alternative Medicine (eCAM) we have the musings of what is appropriate for creating this linkage between the immune system and CAM. 'As a developmental immunobiologist, interested in origins and foundations, I am of course steeped in the beginnings of modern immunology. Let us deal in a bit of musing juxtaposing immunology, science and CAM. We can ask the question: 'Can $e C A M$ Be Scientific? Lessons from Immunology'. According to Burnet (See 4):

The first objective in a serious approach to immunology should be to obtain a broad understanding, with a minimum of detail, of how immunology fits into the pattern of biology - of the way in which the immune system evolved, its function and coordination with other body systems, and its development from the embryo onwards. At the same time, such an outline should provide an adequate background for easy application of immunological ideas to the detail of practical immunological work in public health, clinical, and veterinary practice.

Would it be possible to paraphrase this statement in the context of $e C A M$ so that it reads: 'the first objective in a serious approach to CAM should be to obtain a broad understanding, with a minimum of detail, of how CAM fits into the pattern of biology - of the way in which the neuroendocrineimmune system evolved, its function and coordination with other body systems, and its development from the embryo onwards. At the same time, such an outline should provide an adequate background for easy application of CAM ideas to the detail of practical CAM work in public health, clinical and medical practice, and yet not stray far away from the very biology that under girds it. CAM is organismic, inclusive and not exclusive (4).

Now it is appropriate to turn to the next question. Does any of this fit? So how do we define CAM? Readers may be wondering just what is complementary medicine and what is alternative medicine? Several definitions may be offered based on different points of view and those in turn influenced by professional training, practice and, perhaps, the dictates of funding agencies. The discipline is a heterogeneous subject, to say the least, and it is unlikely that all the adherent disciplines that are sheltered by the umbrella of CAM will carry equal weight or influence or can be subjected to the same rigors of scientific inquiry. Some of these generalizations apply to all disciplines, including those subdisciplines that fall under the aegis of the immunology umbrella. However, perhaps CAM is ripe for an infusion of empiricism. Modern immunology, like CAM, has its earliest roots in concerns for health and disease, but immunology never seemed to have been branded with the aura that often shrouds CAM. There were always a group of experimentalists and, perforce, the necessity to use animal models with which experimentation could be performed to test hypotheses (5).

Once $e C A M$ was launched there was a beginning flurry of works devoted to immunity and CAM. The interesting twist concerned an area that seemed to have been the bridge between what was truly primitive of Metchnikoff's heyday and what is truly immune. Enter therefore and only naturally, the NK cell. According to Takeda and Okumura $(5,6)$ it has been well known since ancient times that CAM, including exercise, provides a lot of benefit to health. Many CAM modalities are believed to prevent or even cure diseases, especially morbid ones such as cancer and aging $(7,8,9,10)$. However, until recently conventional medicine has largely rejected the use of CAM agents because little biological evidence has been provided for the functional mechanisms of many of them. For the past few decades, many modern scientific researchers have rediscovered the value of CAM. Modulation of immune 
functions by CAM agents is the mechanism most widely analyzed and has been suggested to provide some scientific evidence for the biological effects of various CAM agents. Specifically, evidence for up-regulation of natural killer (NK) cell numbers and/or NK cell cytotoxicity by these agents has been accumulating. NK cells have been well established as innate cytotoxic effector cells for self-defense in both vertebrates and invertebrates, in both of which they may use similar mechanisms in the lysis of target cells.

Takeda and Okumura (1) further point out that ever since Macfarlane Burnet proposed the immune surveillance hypothesis against tumor development, the concept has been a hot focus of debate for more than 40 years. The original immune surveillance hypothesis was challenged because nude mice lacking $\mathrm{T}$ cells did not show a higher incidence of cancer than did syngenic immunocompetent mice. However, this contradictory conclusion is now taken as fine evidence that innate immunity is in the critical arms of immune surveillance against tumor development. Moreover, in the innate immune system NK cells, which do not express TCR that recognize specific peptides presented on the major histocompatibility complex (MHC), rather than $\mathrm{T}$ cells, seem well suited for this role. NK cells thus mediating natural cytotoxicity are composed predominantly of large granular lymphocytes (LGL) and some of small agranular lymphocytes, both of which express CD16 and CD56, but no T-cell receptor on the cell surface. NK cells can induce cytolysis in the absence of MHC class I antigen expression on their target cells. This phenomenon is commonly understood according to the 'missing self' hypothesis. Culture of NK cells with some cytokines augments their cytotoxic activity so that they become able to induce cytolysis for a wide spectrum of cells, including tumor cells expressing autologous MHC class I. They are now called lymphokine-activated killer (LAK) cells, although LAK cells are also induced by $\mathrm{T}$ lymphocytes.

\section{Activation of NK Cells by CAM Agents}

For the past few decades, scientific investigations on CAM have remarkably advanced and partly supported their medical efficacy through preclinical and clinical experiments. Here is reviewed briefly information from Takeda and Okumura $(5,6)$. Such CAM modalities include: Oriental medicine, especially traditional Chinese medicine (including Kampo and acupuncture), extract products from natural plants, animal molecules and live lactic acid bacteria. In particular, many investigators have suggested that NK cell activation is one of the critical mechanisms for the biological effects induced by various CAM agents. For example, intake of green tea and some kind of live lactic acid bacteria enhanced NK cell activity. Administration of extracts from ginseng, aged garlic, Viscum album (mistletoe), Cichorium intybus, Echinacea purpurea root, Derris scandens hydroalcholic, some wild plants, Chinese herbs and some kinds of mushrooms significantly augmented NK cytotoxicity or restored NK cell activity in some immune-suppressive conditions. Some of these agents showed inhibition of experimental metastasis of cancer. Oral administration of Phyllanthus emblica, which is known as an excellent source of vitamin $\mathrm{C}$, enhanced NK cell activity and antibody-dependent cellular cytotoxicity (ADCC), thus, supplemental nutriments might enhance NK cell activity. Moreover, acupuncture, skin rubdown, relaxation, massage therapy, music therapy, mirthful laughter and hypnotherapy enhanced NK cell activity and/or NK cell numbers. There are a considerable numbers of studies reporting that acute and chronic exercise and long-term repeated exercise (training effects) elevated NK cell activity in peripheral blood.

\section{Perspectives and Admonitions}

In 1998, the National Center for Complementary and Alternative Medicine (NCCAM) was established by the US Congress at the National Institutes of Health (Bethesda, MD) to investigate CAM modalities rigorously in order to determine which are beneficial and worthy of further consideration for mainstream practice. Among the many CAM approaches that warrant careful investigation are those that claim to sustain, restore or boost immunity. In this review, Goldrosen and Strauss covered the following topics: use of CAM, regulation of CAM, risks of CAM use, clinical trials of CAM, CAM and immunity, dietary supplements and mind-body approaches. They cover some ongoing, large phase III trials of CAM modalities; some CAM modalities that might mediate their effects through the immune system and some herbal products that modulate immune responses. Their Box 1 warns of the challenges of conducting clinical trials of CAM and Figure 1 treats CAM domains and some of the most common examples; a glossary that defines nearly 20 such CAM practices.

Probably one of the greatest salutes to immunologists and practitioners of CAM was the appearance of that paper by Goldrosen and Strauss (3). A pyramid has recently been published in Nature Immunology entitled: 'Complementary and alternative medicine: assessing the evidence for immunological benefits'. In this pyramid, there is a hierarchy of evidence. Information regarding the efficacy and safety of any clinical approach, including those of CAM, spans a continuum that ranges at the base all the way to the peak or the pyramid's point, from anecdotes and retrospective studies to small randomized, controlled trials (phase II clinical trials) and large randomized, controlled trials (phase III clinical trials). In my opinion, this paper and its contents, and the pyramid represent a seminal tribute to the emerging role 
of the immune system in CAM. Moreover, it provides the essential rules necessary for unraveling the tangled threads of legitimate science as approached by $e C A M$ considered to be its mission. Links to the origins of innate immunity that focuses on invertebrates is bridged by the emergence of bioprospecting $(8,9)$ and evidence for clinical trials using products of marine animals (10).

\section{References}

1. Cooper EL, Ru B, Weng N. Earthworms: sources of antimicrobial and anticancer molecules. In: Cooper EL, Yamaguchi N (eds). Complementary and Alternative Approaches to Biomedicine. Kluwer Academic/Plenum Publishers, 2004, 359-390.
2. Cooper EL. What happened in Kanazawa? The birth of eCAM. In: Cooper EL, Yamaguchi N (eds). Complementary and Alternative Approaches to Biomedicine. New York: Kluwer Publishers, 2004.

3. Takeda K, Okumura K. CAM and NK cells. eCAM 2004;1:17-27.

4. Cooper EL. Complementary and alternative medicine, when rigorous, can be science. eCAM 2004;1:1-5.

5. Yamada H. New scientific approach for natural medicine: examples of Kampo medicine. In: Cooper EL, Yamaguchi $\mathrm{N}$ (eds). Complementary and Alternative Approaches to Biomedicine. Kluwer Academic/Plenum Publishers, 2004, 27-34.

6. Cooper EL. Commentary on CAM and NK cells by Kazuyoshi Takeda and Ko Okumura. eCAM 2004;1:29-34.

7. Goldrosen MH, Strauss SE. Complementary and alternative medicine: assessing the evidence for immunological benefits. Nat Rev Immunol 2004;4:912-21.

8. Cooper EL. Bioprospecting: a CAM frontier. eCAM 2005;2:1-3.

9. Cooper EL. Drug discovery, CAM and natural products. eCAM 2004c; $1: 215-7$.

10. Wallace RW. Drugs from the sea: harvesting the results of aeons of chemical evolution. Mol Med Today 1997;3:291-9. 


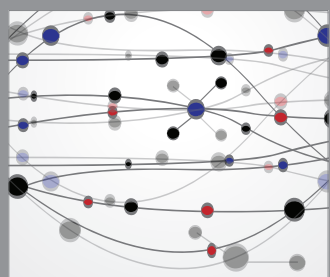

The Scientific World Journal
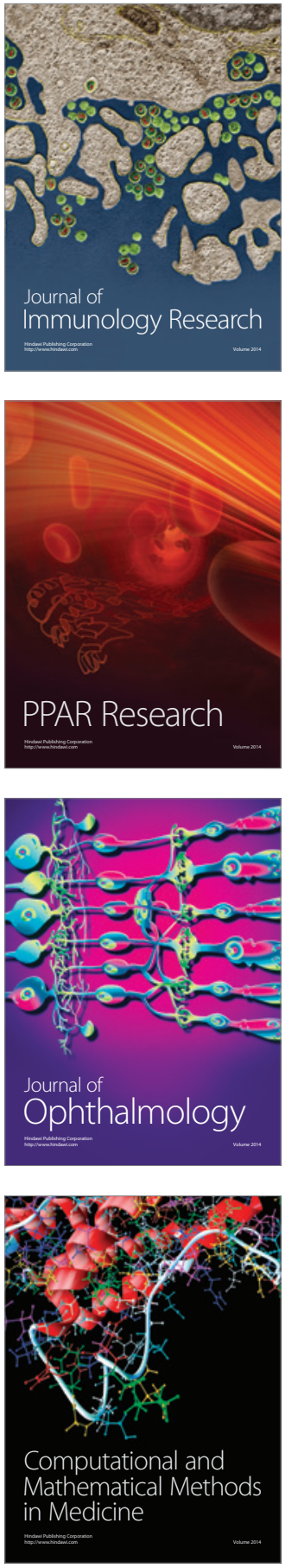

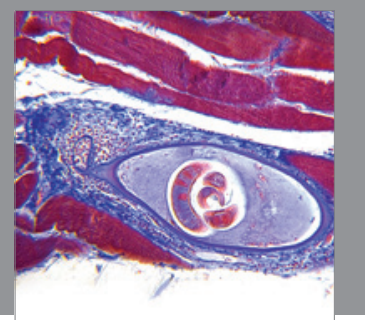

Gastroenterology

Research and Practice
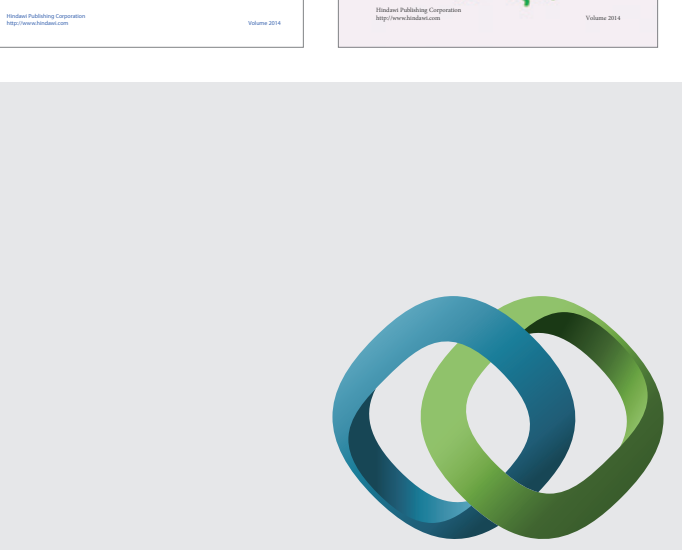

\section{Hindawi}

Submit your manuscripts at

http://www.hindawi.com
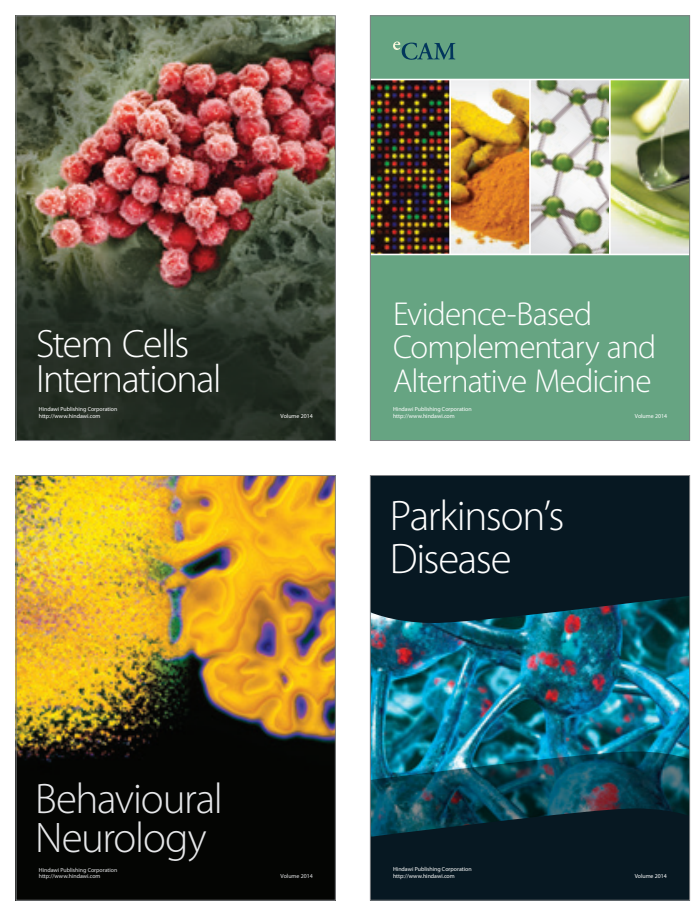

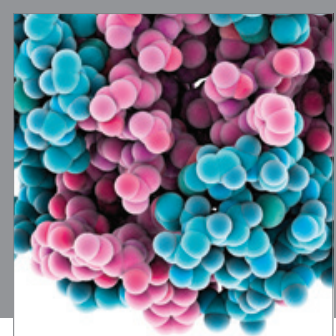

Journal of
Diabetes Research

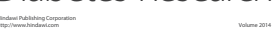

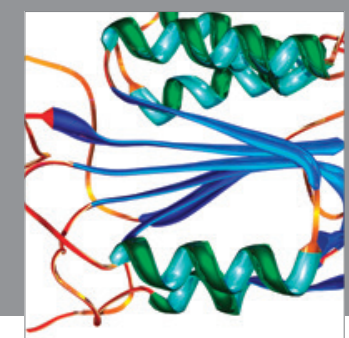

Disease Markers
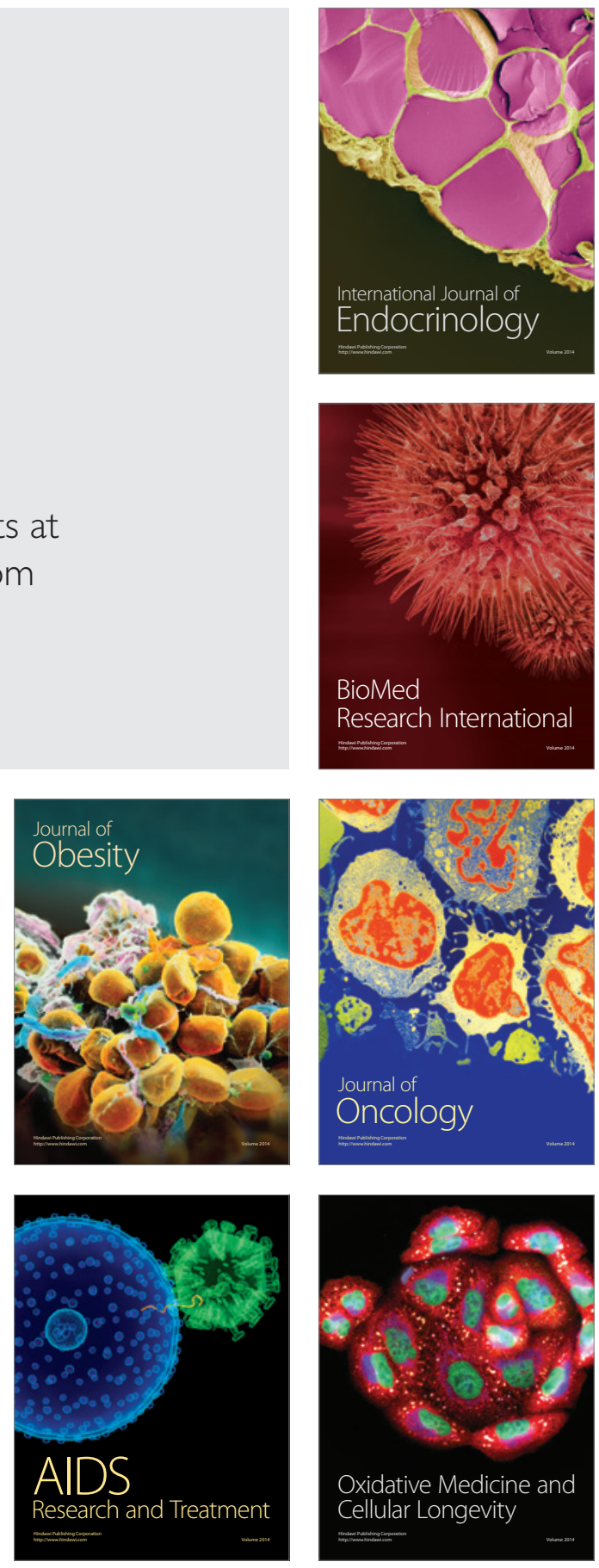